\title{
Women's Questions About Perinatal Cannabis Use and Health Care Providers' Responses
}

\author{
Kelly C. Young-Wolff, PhD, MPH, ${ }^{1}$ Kathleen Gali, $\mathrm{PhD},{ }^{2}$ Varada Sarovar, $\mathrm{PhD}$, \\ Geoffrey W. Rutledge, MD, PhD, ${ }^{3}$ and Judith J. Prochaska, PhD, $\mathrm{MPH}^{2}$
}

\begin{abstract}
Background: Cannabis use is common among individuals of reproductive age. We examined publicly posted questions about perinatal cannabis use and licensed United States health care provider responses.

Materials and Methods: Data were medical questions on perinatal cannabis use posted online from March 2011 to January 2017 on an anonymous digital health platform. Posters were able to "thank" health care providers for their responses and providers could "agree" with other provider responses. We characterized 364 user questions and 596 responses from 277 unique providers and examined endorsement of responses through provider "agrees" and user "thanks."

Results: The most frequent questions concerned prenatal cannabis use detection (24.7\%), effects on fertility (22.6\%), harms of prenatal use to the fetus $(21.3 \%)$, and risks of baby exposure to cannabis through breast milk (14.4\%). Provider sentiment in responses regarding the safety of perinatal cannabis use were coded as $55.6 \%$ harmful, $8.8 \%$ safe, $8.8 \%$ mixed/unsure, and $26.8 \%$ safety unaddressed. Half of providers (49.6\%) discouraged perinatal cannabis use, $0.5 \%$ encouraged use, and $49.9 \%$ neither encouraged nor discouraged use. Provider responses received 1,004 provider "agrees" and 583 user "thanks." Provider responses indicating that perinatal cannabis use is unsafe received more provider "agrees" than responses indicating that use is safe $(B=0.42$, 95\% CI $0.02-0.82, p=0.04)$. User "thanks" did not differ by provider responses regarding safety or dis/ encouragement.

Conclusion: The data indicate public interest in cannabis use effects before, during, and after pregnancy. While most health care providers indicated cannabis use during pregnancy and breastfeeding is not safe, many did not address safety or discourage use, suggesting a missed educational opportunity.
\end{abstract}

Keywords: marijuana, pregnancy, prenatal, postpartum, breast feeding, provider

\section{Introduction}

C ANNABIS USE AND frequency of use are rising among pregnant and reproductive aged women in the United States. ${ }^{1-4}$ While the reasons for increases in perinatal cannabis use are not fully understood, existing data suggest that legalization, easier access, and increasing social acceptability, may play a role. ${ }^{5-8}$ There is evidence that some women use cannabis to alleviate pregnancy-related symptoms (e.g., nausea and vomiting) and to self-medicate symptoms of depression, anxiety, or pain. ${ }^{6-9-15}$ However, due to concerns about health risks, including low infant birthweight and potential impacts on offspring neurodevelopment, ${ }^{16-19}$ the American College of
Obstetricians and Gynecologists currently recommends that pregnant women abstain from using cannabis during pregnancy and while breastfeeding. ${ }^{20}$

An integrative review of women's perceptions of the health effects of prenatal cannabis use found that perceived harms of cannabis use in general are decreasing over time, ${ }^{8}$ and some pregnant women who use cannabis perceive no health risks. ${ }^{5,6}$ Initial studies suggest that clinicians have limited information about the potential risks of perinatal cannabis use,$^{21}$ and often offer little or no response to patient's disclosures of prenatal cannabis use, or focus solely on the potential legal consequences of use rather than providing health information. ${ }^{22}$ Thus, many women express dissatisfaction with health care

\footnotetext{
${ }^{1}$ Division of Research, Kaiser Permanente Northern California, Oakland, California.

${ }^{2}$ Stanford Prevention Research Center, Department of Medicine, Stanford University, Stanford, California.

${ }^{3}$ HealthTap, Mountain View, California.
} 
provider communications about perinatal cannabis use $\mathrm{e}^{8,23}$ and instead search online and seek advice from women who used marijuana in pregnancy. ${ }^{23}$ Meanwhile, cannabis dispensaries and online media tout cannabis as safe for use during pregnancy and effective for treating morning sickness, ${ }^{24,25}$ contributing further to the perception that perinatal cannabis use is without harms.

Telemedicine and virtual health care services offering contact with health care professionals through virtual platforms, including telephones, tablets, social media, and other digital technologies, offer a unique resource for internet users seeking information about perinatal cannabis use. ${ }^{26}$ Digital health communication platforms improve access to primary and specialty care and offer a convenient way to seek medical advice and access information about sensitive topics, oftentimes anonymously. In particular, younger adults, women, and adults with higher levels of education and income are likely to use the internet to seek health or medical information. ${ }^{27,28}$ In research, anonymous online health communication platforms can provide critical insights on public interest and health care provider perspectives and practices. To our knowledge, no prior study has analyzed online health communications related to perinatal cannabis use.

The current study analyzed publicly posted questions on perinatal cannabis use on an online anonymous digital health platform and licensed U.S. health care provider responses. Posters were able to "thank" providers for their responses and providers could "agree" with or endorse other provider responses. We characterized users' questions and provider responses about perinatal cannabis use and examined user satisfaction and provider endorsement of responses through analyzing user "thanks" and provider "agrees."

\section{Materials and Methods}

\section{Study setting and sample}

Data for this study come from HealthTap, an online open digital platform that provides free access to online health information directly sourced from $>140,000$ licensed health care providers across 147 specialties 24 hours a day. Publicly available data, the study qualified for an exemption from the Stanford University IRB.

HealthTap, an open platform available globally through web and mobile devices, was chosen because it is novel, highly utilized, and the data were accessible. HealthTap's health care providers are licensed in the United States and represent each U.S. state. The vast majority of HealthTap users is on the free network side. Anyone, anywhere is able to ask a free question. Those interested in further care can pursue a virtual video consultation with a doctor at a charge.

We utilized the HealthTap repository of publicly available anonymous questions posted online from March 2011 to January 2017 and provider responses. While the questions were posted anonymously, users could indicate their first name, age, and location, a summary of which we received in the aggregate for the question corpus that we analyzed. Data were analyzed in 2019. Our team used a list of cannabisrelated keywords to identify questions related to cannabis (e.g., cannabis, marijuana, weed, tetrahydrocannabinol (THC), cannabidiol (CBD), blunt, bong, ganja, kush, sativa, and cannabinoid). Cannabis-related questions and corresponding provider responses were extracted, data were cleaned to exclude irrelevant questions, and then coded into 13 broad themes. For coding of themes, a defined framework was iteratively developed through team consultation and coding of the corpus. The full dataset included 4,579 questions and 6,891 provider responses. The current study examined 364 questions related to perinatal cannabis use and the corresponding 596 responses from 277 providers. Identifiable names were provided with 204 of the questions, of which 196 (96\%) were traditionally female names, 5 were traditionally male names, and 3 were gender neutral. There were 310 valid birthdates provided, and the average age was 27.6 years $(\mathrm{SD}=9.0)$; $92 \%$ were between the ages of 16 and 45. Of 345 users indicating their location, 331 (96\%) were from the United States and 39 states were represented.

\section{Data coding}

We used directed content analysis methods to code posted questions and provider responses related to perinatal cannabis use. This allowed us to incorporate themes from the literature (e.g., health effects of cannabis use during pregnancy, cannabis detection, legal implications of cannabis use in pregnancy) and provide new insights into emergent themes (e.g., effects on conception, health effects of exposure to cannabis through breast milk, health effects of exposure to second-hand smoke [i.e., smoke from the cigarette or exhaled by the user], or third-hand smoke [i.e., particles and gases left on surfaces in an area where someone has smoked]). The research team iteratively developed and reviewed the coding schemes and coded the questions fully for: (i) stated reasons for cannabis use, and (ii) specific questions for providers. Coding allowed for user questions to fall into multiple categories. Providers could answer more than one question. Responses were coded for valence in addressing the safety of perinatal cannabis use: safe (e.g., "There is no risk here at all'), unsafe (e.g., "Marijuana has been shown to cause a number of health conditions in the fetus"), unsure/mixed (e.g., "I know that's not particularly helpful, but the jury is still out on this one"), and safety not addressed. Responses were also coded for whether providers specifically encouraged or discouraged perinatal cannabis use: encouraged, discouraged use, and neither encouraged nor discouraged use.

To evaluate interrater reliability for coding, Cohen's kappa statistic $^{29}$ and percentage agreement were calculated for a randomly selected $10 \%$ of the questions and provider responses, which were independently double coded by two of the researchers involved in this study. The interrater reliability of coding of the user questions was excellent, with $97.7 \%$ agreement and $\kappa=0.96$. Interrater reliability for coding of provider responses was moderate for perinatal cannabis use safety $(88.1 \%$ agreement, $\kappa=0.79)$ and excellent for dis/encouragement of perinatal cannabis use $(91.5 \%$ agreement, $\kappa=0.83$ ).

\section{Statistical analyses}

We ran descriptive statistics to determine the frequency of user question categories and the stated reasons for cannabis use. Within the question categories, we determined the frequency of provider responses regarding safety and dis/ encouragement of perinatal cannabis use. We tested whether provider responses differed by provider specialty using a 
generalized estimating equation model for multinomial data. We also calculated the percentage of provider responses with at least one user "thank" and at least one provider "agree." Utilizing a generalized linear model with a Poisson distribution and log link we examined the relation between the number of provider "agrees" and user "thanks," after adjusting for the clustering by provider and year of response date to control for time effects (i.e., questions posted longer had more opportunity to accumulate thanks and agrees). Next, we used generalized linear models with a Poisson distribution and log link to examine the relation between the number of user "thanks" and provider "agrees" a response received and provider responses about safety and encouragement of use, incorporating the clustering by provider response and adjusting for year of response date. For these analyses, "thanks" and "agrees" were categorized into 0,1 , 2,3 , and 4 or more and response year was categorized as $2011 / 2012,2013,2014,2015$, and 2016/2017 due to the small number of responses in 2011 and 2017. $p$-Values $<0.05$ (twosided) were considered statistically significant. All analyses were conducted in SAS 9.4.

\section{Results}

\section{Question themes}

The 364 questions related to perinatal cannabis use received 376 content codes (10 questions received 2 content codes, and 1 question received 3 content codes). The questions were categorized into 11 nonmutually exclusive themes that spanned the time period of preconception, prenatal, and postpartum (Table 1). Most prevalent were questions about detection of cannabis in pregnant women or at delivery (24.7\%), effects on the ability to conceive (22.6\%), potential harms of maternal prenatal use to the fetus $(21.3 \%)$, and health effects and exposure to cannabis through breast milk (14.4\%). Questions about effects on ability to conceive most often related to the man $(63.5 \%)$, with $15.3 \%$ relating to the women, and $15.3 \%$ potentially relating to either/both. Less common were questions about legal implications of prenatal cannabis use $(6.4 \%)$, potential harms of second- or third-hand exposure during pregnancy (4.3\%), health effects of secondand third-hand cannabis smoke postpregnancy $(2.7 \%)$, cannabis use after pregnancy ends (1.9\%), harms of cannabis use preconception $(0.8 \%)$, quitting cannabis during pregnancy $(0.5 \%)$, and benefits of prenatal cannabis use $(0.5 \%)$. Few questions $(N=24,6.6 \%)$ stated one or more reasons for perinatal cannabis use, but most commonly stated were to improve appetite or reduce nausea (3.0\%), for general medical conditions $(2.7 \%)$, to improve sleep or increase energy $(1.6 \%)$, and to improve mood $(0.8 \%)$.

\section{Provider responses}

A total of 277 unique doctors (33.2\% primary care physicians, $23.0 \%$ psychiatrists/psychologists, $18.3 \%$ obstetrician/gynecologists, and $25.6 \%$ other) provided 596 responses to users' questions related to perinatal cannabis use with a total of 615 response codes. The 17 additional codes came from provider responses to the 10 double-coded questions and 2 additional codes came from provider responses to the 1 triple-coded question. The mean number of provider responses per question was $2.2(\mathrm{SD}=5.3)$ with a mode and median of $1(\mathrm{IQR}=1)$. Overall, provider responses related to the safety of perinatal cannabis use were coded as $55.6 \%$ harmful, $8.8 \%$ safe, $8.8 \%$ mixed/unsure, and $26.8 \%$ of provider responses did not address safety (Table 2). About half $(49.6 \%)$ specifically discouraged perinatal cannabis use, $0.5 \%$ encouraged use, and $49.9 \%$ neither encouraged nor discouraged use. Responses did not differ by provider specialty, with the exception that providers of "other" specialty were less likely to indicate that prenatal cannabis use was harmful versus safe relative to obstetrician/gynecologists $(B=-1.39,95 \% \mathrm{CI}-2.66$ to $-0.12, p=0.03)$, and psychiatrists/psychologists were more likely to discourage $(B=13.65,95 \%$ CI $11.57-15.73, p<0.001)$ or neither encourage nor discourage $(B=13.43,95 \%$ CI $11.34-15.51$, $p<0.0001$ ) (vs. encourage) prenatal cannabis use relative to obstetrician/gynecologists.

The percentage of provider responses that endorsed perinatal cannabis as harmful ranged from $25.0 \%$ for questions about quitting to $76.9 \%$ for questions about maternal prenatal cannabis use. The percentage of responses that endorsed perinatal cannabis as safe ranged from $0.00 \%$ for questions about quitting and benefits of use during pregnancy to $48.4 \%$ for questions about exposure to second/third-hand smoke during pregnancy. The percentage of provider responses that discouraged perinatal cannabis use ranged from $33.3 \%$ for questions about benefits of use during pregnancy to $100 \%$ for questions about quitting during pregnancy. No providers encouraged cannabis use for any of the question categories except maternal use in pregnancy $(1.4 \%)$ and return to cannabis use after pregnancy $(12.5 \%)$.

\section{User satisfaction and provider consensus}

The 596 provider responses received 1,004 provider "agrees" and 583 user "thanks." The number of provider "agrees" per response ranged from 0 to 12 with a mode of 0 , a median 1, and interquartile range (IQR) $3 ; 60.4 \%$ of provider responses received at least one "agree." The number of user "thanks" per response ranged from 0 to 24 with a mode and median of 0 and IQR of 1; 46.6\% of provider responses received at least one "thanks." More recent provider responses had received fewer provider "agrees" and user "thanks" to date.

Adjusting for clustering by provider and year of response date, provider "agrees" and user "thanks" were positively associated $(B=0.05,95 \%$ CI $0.02-0.08, p=<0.01)$, such that responses that got a greater number of provider "agrees" also received a greater number of user "thanks."

Relative to provider responses that indicated perinatal cannabis use was safe, those that indicated perinatal cannabis use was unsafe received significantly more provider “agrees" $(B=0.42,95 \%$ CI $0.02-0.82, p=0.04)$ (Table 3$)$. Provider agrees did not differ significantly by provider encouragement of perinatal cannabis use, and user "thanks" did not differ significantly by provider responses regarding safety of prenatal cannabis use or by provider encouragement of perinatal cannabis use (Table 3 ).

\section{Discussion}

Our analysis of anonymous online health communications indicates public interest in perinatal cannabis use, particularly in the detection of cannabis use and the potential for 
Table 1. Thematic Coding of Questions Related to Perinatal Cannabis Use

$\begin{array}{lcc} & \mathrm{N}=376,{ }^{\mathrm{a}} & \text { Example quote }(\mathrm{s})\end{array}$

Cannabis exposure before pregnancy $(N=88,23.4 \%)$

1. Conception

Effects of cannabis smoking on ability to conceive

2. Health effects

Potential harms of cannabis use preconception to the pregnancy course, fetus, or the baby

Cannabis exposure during pregnancy $(N=100,26.6 \%)$
3. Health effects of maternal use

Potential harms of cannabis use during pregnancy
$85(22.6)$

"Will smoking pot have an impact on our ability to conceive?"

"Does smoking marijuana make men less fertile?"

"Can smoking marijuana affect female fertility?"

3 (0.8) "Can smoking marijuana before pregnancy cause any complications while pregnancy?"

"If you quit smoking weed years before getting pregnant, can those smoking events deform my future baby?",

$80(21.3)$

"Is it safe to smoke weed in the second trimester?"

"Is vaporizing marijuana during pregnancy bad for the baby"

"Has smoking marijuana ever caused a miscarriage?"

"Can THC itself harm a pregnancy?" "I have smoked weed my entire pregnancy and am almost 30 weeks pregnant. I would like a doctor opinion on if this is a dangerous thing. Should I stop?"

"What could be the effects of eating or smoking marijuana on an unborn baby?'

4. Health effects of Potential harms of second- or second- or third-hand third-hand cannabis smoke cannabis smoke exposure during pregnancy exposure

$16(4.3)$

"Is secondhand marijuana smoke extremely bad for pregnant women to be around?"

"My wife is 4 months pregnant. She was exposed to the second hand marijuana smoke for a few mins, will it effect our unborn child?",

"I am almost 7 weeks pregnant and I am a medical marijuana trimmer. I am wondering if it is safe to work with/around marijuana that has powdery mildew"

5. Quitting cannabis

Treatments to quit cannabis use and managing withdrawal

6. Benefits of cannabis use in pregnancy

Potential benefits of using cannabis during pregnancy

$2(0.5)$

$2(0.5)$

"Can I get meds to quit meth and cannabis when pregnant?",

"My 21 year old sister is pregnant but before that she was smoking weed all day every day while she was pregnant. Could she be going through withdrawal?

"Does weed keep you skinny while pregnant?"

"I am pregnant I have stop smoking weed, I have been getting sick all day everyday and i can't keep nothing down cuz of my pregnancy lose 20 pounds?"

Cannabis exposure postpartum $(N=71,18.9 \%)$

7. Health effects and

Potential harms of breastfeeding after exposure to cannabis cannabis use and duration 1 needs to wait to breastfeed after cannabis use

8. Health effects of Potential harms of second- or third-hand cannabis smoke exposure to the baby

$10(2.7)$ second- or third-hand cannabis smoke exposure
54 (14.4) "Will smoking pot while nursing hurt my baby?' "Does smokin weed while breastfeeding effect the childs development?"

"Is there a way to safely smoke marijuana while breastfeeding?"

"How many times should someone pump and dump after smoking pot so they can continue nursing?"

"I smoked marijuana yesterday. When can I continue to safely breastfeed?"

"Does marijuana smoke hurt babies?'

"Is the smell of marijuana bad for my baby?"

"Is the smell of growing unsmoked marijuana bad for a infant?"

"Should people who smoke weed or cigarettes hold your newborn baby?"

"Is it unsafe to expose a baby to third hand marijuana smoke? (i.e., the smoke absorbed on a persons clothing/skin who just smoked it)"

(continued) 
TABle 1. (CONTINUED)

\begin{tabular}{|c|c|c|c|}
\hline Theme & Description & $\begin{array}{l}\mathrm{N}=376,{ }^{\mathrm{a}} \\
\mathrm{n}(\%)\end{array}$ & Example quote(s) \\
\hline $\begin{array}{l}\text { 9. Cannabis use after } \\
\text { pregnancy ends }\end{array}$ & $\begin{array}{l}\text { Duration } 1 \text { needs to wait } \\
\text { before returning to } \\
\text { cannabis use } \\
\text { postpregnancy (e.g., after } \\
\text { miscarriage, ectopic } \\
\text { pregnancy, cesarean } \\
\text { section) }\end{array}$ & $7(1.9)$ & $\begin{array}{l}\text { "Can you smoke marijuana after surgical abortion?" } \\
\text { "Can I smoke weed during miscarriage?" } \\
\text { "When after a c-section can I smoke weed or drink } \\
\text { alcohol?" }\end{array}$ \\
\hline \multicolumn{4}{|c|}{ Cannabis detection $(N=116,30.9 \%)$} \\
\hline 10. Detection & $\begin{array}{l}\text { Detection of cannabis use in } \\
\text { pregnant woman or in the } \\
\text { baby at delivery and } \\
\text { window of detectability }\end{array}$ & $93(24.7)$ & $\begin{array}{l}\text { "Does marijuana show up in baby poop?" } \\
\text { "How long does THC stay in your and the unborn } \\
\text { babys system, and will they be able to detect it } \\
\text { when I deliver since I quit now?" } \\
\text { "If I don't smoke but am exposed to second hand } \\
\text { marijuana will it be in my babys poor when born?" } \\
\text { "If I smoke marijuana on a daily basis \& being } 22 \\
\text { weeks pregnant, at how many weeks should I stop } \\
\text { before it can be found in my baby's system?", } \\
\text { "I'm } 29 \text { weeks pregnant and have been smoking } \\
\text { marijuana while pregnant. Need to know what I } \\
\text { should do to cleanse my system before birth please } \\
\text { help." }\end{array}$ \\
\hline 11. Legal implications & $\begin{array}{l}\text { Legal implications of testing } \\
\text { positive or using during } \\
\text { pregnancy }\end{array}$ & $24(6.4)$ & $\begin{array}{l}\text { "If I smoked weed in my pregnancy an but im clean } \\
\text { when I go into labor will cps still get involved if its } \\
\text { not in mine or the babies system?" } \\
\text { "I quit smoking pot but am worried I will still test } \\
\text { positive at my first prenatal appointment for THC. } \\
\text { Will I get into trouble for this?" } \\
\text { "I live in Chicago and I smoke weed during my } \\
\text { pregnancy would they as in CPS take my baby } \\
\text { away from me?" } \\
\text { "Will the doctor test my baby at her checkups and } \\
\text { take her if she test positive?" }\end{array}$ \\
\hline
\end{tabular}

\footnotetext{
${ }^{a}$ Questions could fall into multiple categories. There were 364 questions and 376 codes (10 questions were double coded and 1 was triple coded).

THC, tetrahydrocannabinol.
}

health harm to the fetus and baby. Health concerns of greatest interest are related to fertility, fetal prenatal effects, and cannabis exposure to the infant through breast milk. Less common were questions about legal implications of use, potential harms of second- or third-hand cannabis smoke exposure, use after termination of a pregnancy, and fetal harms of cannabis use preconception. Very rare were inquiries about quitting during pregnancy and potential benefits of prenatal cannabis use. The findings are consistent with prior qualitative studies indicating that women desire health information on the use of cannabis during pregnancy and when breastfeeding. ${ }^{23}$

While few of the questions mentioned reasons for perinatal cannabis use, those that did referred to effects of enhancing appetite or reducing nausea, improving sleep or increasing energy, improving mood, and use to treat general medical conditions. Findings support recent studies indicating that pregnant women may use cannabis to self-medicate pregnancy-related symptoms and to improve mood, perhaps in part due to messages from online media and cannabis retailers recommending cannabis to treat morning sickness and endorsing the safety of cannabis use in pregnancy. ${ }^{24,25}$

Analysis of provider responses yields important insight into how clinicians communicate about perinatal cannabis use. Among all provider responses, just over half indicated that perinatal cannabis use is harmful, and half specifically discouraged perinatal cannabis use. Providers were most likely to indicate that perinatal cannabis use was harmful to fertility and pregnancy or when breastfeeding and were most likely to discourage cannabis use in pregnancy or when breastfeeding. Providers were least likely to indicate that cannabis was harmful in responding to questions about quitting and second- or thirdhand smoke exposures. Providers, however, were most likely to encourage quitting in response to questions asking about quitting. Providers were least likely to discourage use in the context of questions about the benefits of cannabis use, impacts on fertility, and exposure to second- or third-hand smoke.

Overall, there was substantial variability in provider responses regarding perinatal cannabis use harms and discouragement of use, suggesting the need for medical training and education on this topic and dissemination of clinical practice guidelines. Of note, obstetrician/gynecologists emphasized the harms of perinatal cannabis more than providers of "other" specialties, whereas psychiatrists/psychologists were more likely to discourage versus encourage prenatal cannabis use relative to obstetrician/gynecologists. It is possible that providers understand the potential risks but chose not to address harms 
Table 2. Provider Responses $(N=615)$ to Questions Concerning Perinatal Cannabis Use

\begin{tabular}{|c|c|c|c|c|c|c|c|c|}
\hline & \multirow{2}{*}{$\begin{array}{c}\text { Provider } \\
\text { responses, } \\
\mathrm{N}(\%)\end{array}$} & \multicolumn{4}{|c|}{$\begin{array}{l}\text { Provider responses regarding safety } \\
\text { of cannabis uselexposure, } \mathrm{n}(\%)^{\mathrm{a}}\end{array}$} & \multicolumn{3}{|c|}{$\begin{array}{l}\text { Provider responses encouraging } \\
\text { or discouraging uselexposure, } \mathrm{n}(\%)\end{array}$} \\
\hline & & Harmful & Safe & $\begin{array}{l}\text { Mixed/ } \\
\text { Unsure }\end{array}$ & $\begin{array}{c}\text { Not } \\
\text { addressed }\end{array}$ & Discourage & Encourage & $\begin{array}{c}\text { Not } \\
\text { addressed }\end{array}$ \\
\hline Total & $615(100)$ & $342(55.6)$ & $54(8.8)$ & $54(8.8)$ & $165(26.8)$ & 305 (49.6) & $3(0.5)$ & $307(49.9)$ \\
\hline \multicolumn{9}{|l|}{ Question category } \\
\hline \multicolumn{9}{|l|}{ Exposure before pregnancy } \\
\hline Ability to conceive & $130(21.8)$ & $86(66.2)$ & $18(13.9)$ & $11(8.5)$ & $15(11.5)$ & $45(34.6)$ & $0(0)$ & $85(65.4)$ \\
\hline Health effects & $6(1.0)$ & $3(50.0)$ & $1(16.7)$ & $2(33.3)$ & $0(0)$ & $3(50.0)$ & $0(0)$ & $3(50.0)$ \\
\hline \multicolumn{9}{|c|}{ Exposure during pregnancy } \\
\hline Maternal use & $143(24.0)$ & $110(76.9)$ & 7 (4.9) & $14(9.8)$ & $12(8.4)$ & $82(57.3)$ & $2(1.4)$ & $59(41.3)$ \\
\hline $\begin{array}{l}\text { Second/Third-hand } \\
\text { smoke }\end{array}$ & $31(5.2)$ & $8(25.8)$ & $15(48.4)$ & $5(16.1)$ & $3(9.7)$ & $13(41.9)$ & $0(0)$ & $18(58.1)$ \\
\hline Quitting use & $4(0.7)$ & $1(25.0)$ & $0(0)$ & $0(0)$ & $3(75.0)$ & $4(100)$ & $0(0)$ & $0(0)$ \\
\hline Benefits of use & $3(0.5)$ & $1(33.3)$ & $0(0)$ & $0(0)$ & $2(66.7)$ & $1(33.3)$ & $0(0)$ & $2(66.7)$ \\
\hline \multicolumn{9}{|l|}{ Exposure postpartum } \\
\hline Use and breastfeeding & 99 (16.6) & $63(63.6)$ & $3(3.0)$ & $10(10.1)$ & $23(23.2)$ & $65(65.7)$ & $0(0)$ & $34(34.3)$ \\
\hline $\begin{array}{l}\text { Second/Third-hand } \\
\text { smoke }\end{array}$ & $19(3.2)$ & $11(57.9)$ & $3(15.8)$ & $3(15.8)$ & $2(10.5)$ & $7(36.8)$ & $0(0)$ & $12(63.2)$ \\
\hline Return to use & $8(1.3)$ & $3(37.5)$ & $1(12.5)$ & $2(25.0)$ & $2(25.0)$ & $4(50.0)$ & $1(12.5)$ & $3(37.5)$ \\
\hline \multicolumn{9}{|l|}{ Cannabis detection } \\
\hline Detection & $135(22.7)$ & 43 (31.9) & $5(3.7)$ & $7(5.2)$ & $80(59.3)$ & $64(47.4)$ & $0(0)$ & $71(52.6)$ \\
\hline Legal implications & $37(6.2)$ & $13(35.1)$ & $1(2.7)$ & $0(0)$ & $23(62.2)$ & $17(46.0)$ & $0(0)$ & $20(54.1)$ \\
\hline
\end{tabular}

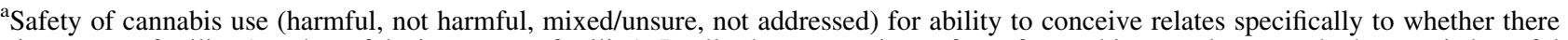
are impacts on fertility (i.e., harmful=impacts on fertility). In all other categories, safety of cannabis use relates to whether use is harmful during pregnancy and postpartum. Provider responses could address questions that fell into multiple categories. There were 596 unique provider responses and 615 response codes (17 additional codes came from provider responses to the 10 double-coded questions and 2 additional codes came from provider responses to the 1 triple-coded question).

or discourage use out of discomfort or concern of appearing judgmental or stigmatizing. Yet, users' thanks of provider responses did not differ by mention of harms or dis/encouragement of use, suggesting that users value provider responses even when they indicate that cannabis use is unsafe

Table 3. Results from Adjusted Multilevel Analyses of the Relation Between Provider RESPONSES TO QUESTIONS AND "AGREES" AND "THANKS"

\begin{tabular}{lcc}
\hline Provider responses & Beta $(95 \%$ CI $)$ & $\mathrm{p}$ \\
\hline Provider agrees & & \\
Safety of perinatal cannabis use & \\
Not discussed & $0.22(-0.24$ to 0.69$)$ & 0.3490 \\
Mixed & $0.21(-0.36$ to 0.77$)$ & 0.4708 \\
Unsafe & $0.42(0.02$ to 0.82$)$ & 0.0391 \\
Safe & Reference & Reference \\
Encouragement of perinatal cannabis use & \\
Not addressed & $1.61(-0.24$ to 3.45$)$ & 0.0883 \\
Discourage & 1.77 (-0.06 to 3.62$)$ & 0.0582 \\
Encourage & Reference & Reference \\
User thanks & & \\
Safety of perinatal cannabis use & \\
Not discussed & $0.06(-0.42$ to 0.54$)$ & 0.7976 \\
Mixed & 0.30 (-0.16 to 0.75$)$ & 0.2017 \\
Unsafe & 0.04 (-0.36 to 0.43$)$ & 0.8496 \\
Safe & Reference & Reference \\
Encouragement of perinatal cannabis use & \\
Not addressed & 0.54 (-0.65 to 1.73$)$ & 0.3773 \\
Discourage & 0.44 (-0.76 to 1.64$)$ & 0.4705 \\
Encourage & Reference & Reference \\
\hline
\end{tabular}

CI, confidence interval. or discourage its use. In contrast, provider responses that indicated perinatal cannabis use was unsafe received significantly more provider "agrees" than provider responses that indicated cannabis use was safe, suggesting some consensus among providers about potential health risks.

To date, 11 states have legalized cannabis for recreational use and 34 states and the District of Columbia have legalized cannabis use for medical use. ${ }^{30}$ Support for cannabis legalization in the United States is rising, with the majority of U.S. adults of reproductive age favoring cannabis legalization in $2018 .^{31}$ Notably, a study in Maryland found that $62 \%$ of pregnant women who used cannabis during pregnancy reported that they would increase their use if it were legalized. ${ }^{6}$ As legalization of recreational cannabis use spreads, timely and objective clinical advice on the potential health risks of perinatal cannabis use are needed to help people make informed decisions about use. While additional research is needed to better understand the specific health risks associated with frequency of prenatal cannabis use and mode of cannabis administration (e.g., smoking, vaping, use of edibles), enough is known about the potential impacts on fetal growth and neurodevelopment for national guidelines to strongly advise women to discontinue cannabis use during pregnancy and while breastfeeding. ${ }^{20}$

Health care providers can play a critical role in reducing risks by providing education to women of reproductive age about the potential harms of cannabis use during pregnancy and while breastfeeding, advice to quit, and information about medically recommended treatments for pregnancy-related symptoms. In addition, while limited, current research indicates that cannabis use may negatively impact male fertility, ${ }^{32}$ and providers can convey this information. That many of the provider responses 
analyzed in the current study were coded as reflecting mixed messages/uncertainty about whether perinatal cannabis use is safe or did not address safety at all, and many did not explicitly discourage use, represents a missed opportunity for education and advice and may, through omission, encourage use. Research has shown that when health care providers do not offer consultation or education and specific recommendations about prenatal cannabis use during routine prenatal visits, patients perceive this to indicate that prenatal cannabis use is safe. ${ }^{23}$

\section{Limitations}

Study results should be interpreted in the context of several limitations. First, HealthTap user questions were anonymous, and only limited user characteristics (e.g., age, sex, location) were available, in the aggregate, and not for the full sample. Hence, we are unable to determine if the results generalize to any specific population. Users may have posted more than one question, and we could not adjust this in our analyses due to the anonymous nature of the data. In contrast, provider responses were publicly available with identifying information, which may have influenced how providers responded. Furthermore, providers who choose to answer online questions related to perinatal cannabis use may not be representative of all providers.

\section{Conclusion}

As the acceptability and accessibility of cannabis increases, ${ }^{31,33}$ and cannabis use rises before, during, and after pregnancy, ${ }^{1-4}$ medical providers need training and education to answer questions about cannabis use during the perinatal period. Results from this study highlight pertinent questions about perinatal cannabis use, including detection, safety, and impact on fertility, and indicate that people are seeking information online from health care professionals. While most providers indicated that use during pregnancy and breastfeeding is not safe, and discouraged use consistent with national guidelines ${ }^{20}$ many providers did not address harms or discourage use, suggesting a missed opportunity. Additional timely and objective data on the health risks of perinatal cannabis use are needed to help individuals to make informed decisions as more states legalize cannabis for medical and recreational use. In the meantime, providers can reduce potential harms through education and advice to discontinue marijuana use during pregnancy and while breastfeeding.

\section{Author Disclosure Statement}

G.R. is Chief Medical Officer and Co-Founder of HealthTap. All other authors have no financial conflicts of interest.

\section{Funding Information}

This study was supported by a NIH NIDA K01 Award (DA043604) and NHLBI T32 Award (HL007034-43).

\section{References}

1. Brown QL, Sarvet AL, Shmulewitz D, Martins SS, Wall MM, Hasin DS. Trends in marijuana use among pregnant and nonpregnant reproductive-aged women, 2002-2014. JAMA 2017;317:207-209.

2. Young-Wolff KC, Tucker LY, Alexeeff S, et al. Trends in self-reported and biochemically tested marijuana use among pregnant females in California from 2009-2016. JAMA 2017;318:2490-2491.

3. Young-Wolff KC, Sarovar V, Tucker LY, et al. Selfreported daily, weekly, and monthly cannabis use among women before and during pregnancy. JAMA Netw Open 2019;2:e196471.

4. Volkow ND, Han B, Compton WM, McCance-Katz EF. Selfreported medical and nonmedical cannabis use among pregnant women in the United States. JAMA 2019;322:167-169.

5. Ko JY, Farr SL, Tong VT, Creanga AA, Callaghan WM. Prevalence and patterns of marijuana use among pregnant and nonpregnant women of reproductive age. Am J Obstet Gynecol 2015;213:201.e1-201.e10.

6. Mark K, Gryczynski J, Axenfeld E, Schwartz RP, Terplan $M$. Pregnant women's current and intended cannabis use in relation to their views toward legalization and knowledge of potential harm. J Addict Med 2017;11:211-216.

7. Crume TL, Juhl AL, Brooks-Russell A, Hall KE, Wymore $\mathrm{E}$, Borgelt LM. Cannabis use during the perinatal period in a state with legalized recreational and medical marijuana: The association between maternal characteristics, breastfeeding patterns, and neonatal outcomes. J Pediatr 2018;197:90-96.

8. Bayrampour $\mathrm{H}$, Zahradnik $\mathrm{M}$, Lisonkova S, Janssen P. Women's perspectives about cannabis use during pregnancy and the postpartum period: An integrative review. Prev Med 2019;119:17-23.

9. Chang JC, Tarr JA, Holland CL, et al. Beliefs and attitudes regarding prenatal marijuana use: Perspectives of pregnant women who report use. Drug Alcohol Depend 2019;196: 14-20.

10. Driscoll KE, Sit DKY, Moses-Kolko EL, et al. Mood symptoms in pregnant and postpartum women with bipolar disorder: A naturalistic study. Bipolar Disord 2017;19:295304.

11. Mark K, Desai A, Terplan M. Marijuana use and pregnancy: Prevalence, associated characteristics, and birth outcomes. Arch Womens Ment Health 2016;19:105-111.

12. Oh S, Salas-Wright CP, Vaughn MG, DiNitto DM. Marijuana use during pregnancy: A comparison of trends and correlates among married and unmarried pregnant women. Drug Alcohol Depend 2017;181:229-233.

13. Young-Wolff KC, Adams SR, Klebaner D, et al. Evaluating the impact of eliminating copayments for tobacco cessation pharmacotherapy. Med Care 2018;56:912-918.

14. Young-Wolff KC, Sarovar V, Tucker LY, et al. Trends in marijuana use among pregnant women with and without nausea and vomiting in pregnancy, 2009-2016. Drug Alcohol Depend 2019;196:66-70.

15. Roberson EK, Patrick WK, Hurwitz EL. Marijuana use and maternal experiences of severe nausea during pregnancy in Hawai'i. Hawaii J Med Public Health 2014;73:283-287.

16. Metz TD, Allshouse AA, Hogue CJ, et al. Maternal marijuana use, adverse pregnancy outcomes, and neonatal morbidity. Am J Obstet Gynecol 2017;217:478.e471-478.e478.

17. Sharapova SR, Phillips E, Sirocco K, Kaminski JW, Leeb RT, Rolle I. Effects of prenatal marijuana exposure on neuropsychological outcomes in children aged 1-11 years: A systematic review. Paediatr Perinat Epidemiol 2018;32: 512-532.

18. National Academies of Sciences Engineering and Medicine, Health and Medicine Division. The health effects of cannabis and cannabinoids. The current state of evidence and recommendations for research. National Academy of Sciences. Published 2017. Updated January. Available at: 
http://nationalacademies.org/hmd/ /media/Files/Report $\%$ 20Files/2017/Cannabis-Health-Effects/Cannabis-reporthighlights.pdf Accessed September 1, 2019.

19. Ryan SA, Ammerman SD, O'Connor ME, Committee on Substance Use, Prevention, Section on Breatfeeding. Marijuana use during pregnancy and breastfeeding: Implications for neonatal and childhood outcomes. Pediatrics 2018; 142:e20181889.

20. Committee on Obstetric Practice. Committee opinion no. 722: Marijuana use during pregnancy and lactation. Obstet Gynecol 2017;130:e205-e209.

21. Holland CL, Nkumsah MA, Morrison P, et al. "Anything above marijuana takes priority": Obstetric providers' attitudes and counseling strategies regarding perinatal marijuana use. Patient Educ Couns 2016;99:1446-1451.

22. Holland CL, Rubio D, Rodriguez KL, et al. Obstetric health care providers' counseling responses to pregnant patient disclosures of marijuana use. Obstet Gynecol 2016;127:681687.

23. Jarlenski M, Tarr JA, Holland CL, Farrell D, Chang JC. Pregnant women's access to information about perinatal marijuana use: A qualitative study. Womens Health Issues 2016;26:452-459.

24. Jarlenski M, Koma JW, Zank J, Bodnar LM, Tarr JA, Chang JC. Media portrayal of prenatal and postpartum marijuana use in an era of scientific uncertainty. Drug Alcohol Depend 2018;187:116-122.

25. Dickson B, Mansfield C, Guiahi M, et al. Recommendations from cannabis dispensaries about first-trimester cannabis use. Obstet Gynecol 2018;131:1031-1038.

26. Nochomovitz M, Sharma R. Is it time for a new medical specialty?: The medical virtualist. JAMA 2018;319:437-438.

27. Powell JA, Darvell M, Gray JA. The doctor, the patient and the world-wide web: How the internet is changing healthcare. J R Soc Med 2003;96:74-76.
28. Hesse BW, Nelson DE, Kreps GL, et al. Trust and sources of health information: The impact of the Internet and its implications for health care providers: Findings from the first Health Information National Trends Survey. Arch Intern Med 2005;165:2618-2624.

29. McHugh ML. Interrater reliability: The kappa statistic. Biochem Med (Zagreb) 2012;22:276-282.

30. National Conference of State Legislatures. State medical marijuana laws. Published 2019. Updated March 5. Available at: www.ncsl.org/research/health/state-medical-marijuanalaws.aspx Accessed September 1, 2019.

31. Hartig H, Geiger A. About six-in-ten Americans support marijuana legalization. Pew Research Center web site. Published 2018. Updated October 8. Available at: www .pewresearch.org/fact-tank/2018/10/08/americans-supportmarijuana-legalization Accessed September 1, 2019.

32. Payne KS, Mazur DJ, Hotaling JM, Pastuszak AW. Cannabis and male fertility: A systematic review. J Urol 2019; 202:674-681.

33. Azofeifa A, Mattson ME, Schauer G, McAfee T, Grant A, Lyerla R. National estimates of marijuana use and related indicators-National Survey on Drug Use and Health, United States, 2002-2014. MMWR Surveill Summ 2016; $65: 1-28$.

Address correspondence to: Kelly C. Young-Wolff, PhD, $M P H$ Division of Research Kaiser Permanente Northern California 2000 Broadway Oakland, CA 94612

E-mail: kelly.c.young-wolff@kp.org 